\title{
Advances in Open Charm Physics at CLEO-c
}

\author{
Paras Naik ${ }^{\mathrm{a}}$

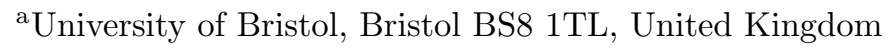

We present a survey of CLEO-c open charm results. The unprecedented full data sample includes over 10 million $D$ mesons and approximately 1.2 million $D_{s}$ mesons. These results substantially extend the reach and understanding of heavy flavor physics. The world community will benefit as results from CLEO-c extend the reach of the Belle experiment at KEK and the LHCb experiment at CERN and lay foundations for the physics program of the BESIII experiment in China.

\section{Introduction}

The CLEO-c experiment [1] at the CESR-c (Cornell Electron Storage Ring - charm) $e^{+} e^{-}$ collider has collected large data samples in the charm threshold energy region. Open charm samples have allowed the CLEO-c experiment to have an impact on measurements of the CabibboKobayashi-Maskawa (CKM) matrix elements, improved our knowledge of hadronic $D$ and $D_{s}$ decays, and enabled studies of rare charm processes.

Precision measurements of the weak phases that compose the unitarity triangle $(\alpha, \beta$, and $\gamma$ ) allow us to test the internal consistency of the CKM model and search for signatures of New Physics. The CKM phase $\gamma$ is only constrained by direct measurements to $\left(71_{-25}^{+21}\right)^{\circ}[2]$. The most promising methods of determining the CKM phase $\gamma$ exploit the interference within $B^{\mp} \rightarrow D K^{\mp}$ decays, where the neutral $D$ meson is a $D^{0}$ or $\bar{D}^{0}$. The most straightforward of these strategies considers two-body final states of the $D$ meson, but additional information can be gained from strategies that consider multi-body final states. The parameters associated with the specific final states needed for these analyses can be extracted from correlations within CLEO-c $\psi(3770)$ data.

Lattice QCD theory offers the prospect of a systematically-improvable method of calculating hadronic properties from first principles. In the past decade, theoretical and technological advances have allowed Lattice QCD to provide pre- cise predictions that estimate systematic uncertainties reliably. An exciting application of these results allows the extraction of electroweak parameters in the $B$ meson system. However, it is desirable to test them elsewhere, for example in the charm system, and CLEO-c provides excellent data for these tests.

\section{Detector and Data Samples}

The CLEO-c detector was a symmetric general purpose detector that provided excellent electromagnetic calorimetry, charged particle tracking and identification, and near $4 \pi$ solid angular coverage. The experiment is described in detail elsewhere [1].

The relevant data sets for the following analyses were collected at center of mass energies of approximately $3.77 \mathrm{GeV}$ (the peak of the $\psi(3770)$ resonance) and $4.17 \mathrm{GeV}$. The former data set is used for $D^{0}$ and $D^{+}$analyses, and the latter for $D_{s}$ physics. Except where noted, all analyses use the full CLEO-c data sets of $818 \mathrm{pb}^{-1}$ at 3.77 $\mathrm{GeV}$ and $600 \mathrm{pb}^{-1}$ at $4.17 \mathrm{GeV}$.

At $3.77 \mathrm{GeV}$ the only allowed open charm final states are $D^{0} \bar{D}^{0}$ and $D^{+} D^{-}$. At $4.17 \mathrm{GeV}$ the only allowed states involving a $D_{s}$ meson are $D_{s}^{+} D_{s}^{-}$and $D_{s}^{ \pm} D_{s}^{* \mp}$. Since only two $D$ mesons of opposite flavor are produced, we may use the double tagging technique pioneered by Mark III [3]. This allows CLEO-c to obtain very clean samples of charm meson decays, with minimal combinatoric background. In particular, using neutral $D$ 
meson tags allow us to exploit quantum correlations of the initial state $\left(\psi(3770) \rightarrow D^{0} \bar{D}^{0}\right)$. Also, neutrinos and $K_{L}$ mesons may be inferred via the full reconstruction of the observed particles in an event.

\section{Strong Phases for $\gamma / \phi_{3}$ Measurements}

\section{1. $D \rightarrow K^{-} \pi^{+}$}

Atwood, Dunietz and Soni (ADS) 4 have suggested considering $D$ decays to non- $C P$ eigenstates as a way of maximizing sensitivity to $\gamma$ measured via studies of the rates of $B^{\mp} \rightarrow D K^{\mp}$ decays. $D$ meson final states such as $K^{-} \pi^{+}$, which may arise from either a Cabibbo favored $D^{0}$ decay or a doubly Cabibbo suppressed $\bar{D}^{0}$ decay, can lead to large interference effects and hence provide particular sensitivity to $\gamma$. The interference phase, $\delta_{D}^{K \pi}$, also relates the $D^{0}$ mixing parameters $y$ and $y^{\prime}$. The differences in the effective branching fraction for $K^{-} \pi^{+}$decay opposite $C P$-even and -odd eigenstates, semileptonic decays, and $K^{+} \pi^{-}$are sensitive to both $\delta_{D}^{K \pi}$ and to $D^{0}$ mixing parameters. With $281 \mathrm{pb}^{-1}$ of data, CLEO-c has measured $\delta_{D}^{K \pi}$ to be $\left(22_{-15}^{+14}\right)^{\circ}$ through a quantum correlated analysis of completely reconstructed $\psi(3770) \rightarrow D \bar{D}$ decays [5].

\subsection{Multi-body Decays}

The ADS formalism can be extended by considering multi-body decays of the $D$ meson. However, a multi-body $D$-decay amplitude is potentially different at any point within the decay phase space, because of the contribution of intermediate resonances. It is shown in Ref. 6] how the rate equations for the two-body ADS method should be modified for use with multibody final states. The result is an effective average phase and a "coherence factor" which reflects the dilution of total interference relative to the expectation for a simple two-body decay. CLEOc has measured these for the $D^{0} \rightarrow K^{-} \pi^{+} \pi^{0}$ and $D^{0} \rightarrow K^{-} \pi^{+} \pi^{+} \pi^{-}$decays, observing significant coherence in the former [7].

3.3. $K_{S, L}^{0} \pi^{+} \pi^{-}$and $K_{S, L}^{0} K^{+} K^{-}$

Dalitz plot analyses of the three-body decay $D \rightarrow K_{S}^{0} \pi^{+} \pi^{-}$together with studies of $B^{\mp} \rightarrow$ $D K^{\mp}$ processes currently provide the best mea- surements of the CKM weak phase $\gamma$ 89. However, $D \rightarrow K_{S}^{0} \pi^{+} \pi^{-}$Dalitz plot analyses are sensitive to the choice of the model used to describe the three-body decay, which currently introduces a model systematic uncertainty on the determination of $\gamma$ which is greater than $5^{\circ}[9$. For $\mathrm{LHCb}$ and future Super- $B$ factories, this uncertainty will become a major limitation. A model independent approach to understanding the $D$ decay has been proposed by Giri and further investigated by Bondar [10, which takes advantage of the quantum correlated $D^{0} / \bar{D}^{0}$ CLEO-c data produced at the $\psi(3770)$ resonance.

CLEO-c has performed this measurement for the $K_{S, L}^{0} \pi^{+} \pi^{-}$decay [11] and for the $K_{S, L}^{0} K^{+} K^{-}$ mode [12] which may also be used in this method. Fig. 1 shows the effect of the $C P$ correlations on the $D^{0} \rightarrow K_{S}^{0} \pi^{+} \pi^{-}$Dalitz plot. Up to small effects $K_{L}^{0} \pi^{+} \pi^{-}$has a $C P$ structure opposite that of $K_{S}^{0} \pi^{+} \pi^{-}$, and similarly for $K_{L}^{0} K^{+} K^{-}$and $K_{S}^{0} K^{+} K^{-}$.

\subsection{Impact on $\gamma$ Measurement}

The $D^{0} \rightarrow K_{S}^{0} \pi^{+} \pi^{-}$analysis is expected to reduce the current $7-9^{\circ}$ model uncertainties from BaBar and Belle measurements 89 to around $2^{\circ}$ [11]3 at $\mathrm{LHCb}$. The $K^{-} \pi^{+}$and multi-body coherence factor measurements are projected to improve the precision of $\mathrm{LHCb}$ in $B \rightarrow D K$ by 8-35\% (depending on unknown $B$ decay parameters) to $2.2-3.5^{\circ}$ after $10 \mathrm{fb}^{-1}$ of $\mathrm{LHCb}$ data taking [14.

\section{Leptonic Decays and Decay Constants}

The decay $X^{+} \rightarrow \ell^{+} \nu$ of pseudoscalar $X$ involves a hadronic current (parametrized by the single "decay constant" $\left.f_{X}\right)$ and a wellunderstood Standard Model leptonic current. The branching fraction can be written as:

$\mathcal{B}\left(X^{+} \rightarrow \ell^{+} \nu\right)=f_{X}^{2}|V|^{2} \frac{G_{F}^{2}}{8 \pi} m_{X} m_{\ell}^{2}\left(1-\frac{m_{\ell}^{2}}{m_{X}^{2}}\right)^{2}$

where $V$ is the relevant element of the CKM matrix connecting the valence quarks of $X\left(V_{c d}\right.$ and $V_{c s}$ for $D^{+}$and $D_{s}^{+}$, respectively). The decay constant is essentially a measure of the wave function of the meson at zero separation between the 
quarks. This makes the decay constant relevant for processes where the relevant length scales are much smaller than the hadron size, including the loop diagrams for $B_{d}^{0}$ and $B_{s}^{0}$ mixing (our primary source of information on $V_{t d}$ ).

We can determine $f_{X}^{2}|V|^{2}$ by measuring the branching fraction of $X$. Thus either the decay constant or the CKM element can be determined if we know the other quantity. CLEO-c has measured the $D^{+}$and $D_{s}^{+}$decay constants in multiple decay modes. The results of all CLEO-c leptonic branching fraction measurements and the corresponding decay constants are shown in Table 1 . The values of input parameters used to obtain these values are listed in the corresponding papers 15161718 .

\section{Exclusive Semileptonic Decays}

Exclusive semileptonic decays are more difficult to parameterize than leptonic decays since there more than two particles in the final state. The partial width for the decay $X \rightarrow X^{\prime} \ell \nu$, where $X$ and $X^{\prime}$ are pseudoscalars, can be written as:

$$
\frac{d \Gamma\left(X \rightarrow X^{\prime} \ell \nu\right)}{d q^{2}}=\frac{G_{F}^{2}}{24 \pi^{3}}\left[f_{+}^{X \rightarrow X^{\prime}}\left(q^{2}\right)|V|\right]^{2} p_{X}^{3}
$$

in the limit where the charged lepton mass is negligible. In Equation 2, $q^{2}$ is the invariant mass squared of the $\ell \nu$ system, $|V|$ is the relevant CKM matrix element for the weak transition, and $f_{+}^{X \rightarrow X^{\prime}}$ is a form factor which represents the hadronic physics interactions. As in the leptonic decay case, input for either $|V|$ or $f_{+}$ allows determination of the other. We analyze $D \rightarrow(K, \pi) e^{+} \nu$ decays 19 in order to determine the form factors in bins of $q^{2}$. Reasonable agreement on the form factor shape and normalization is found with a lattice QCD prediction from the FNAL, MILC, and HPQCD collaborations [20]. A comparison of our results to the theory can be seen in Figure 2 Using lattice predictions for $\left|f_{+}(0)\right|$, values for $\left|V_{c d}\right|$ and $\left|V_{c s}\right|$ are also obtained, which are limited by lattice uncertainties.

\section{REFERENCES}

1. Y. Kubota et al., Nucl. Instrum. Meth. Phys. Res., Sect. A 320, 66 (1992);
D. Peterson et al., Nucl. Instrum. Meth. Phys. Res., Sect. A 478, 142 (2002).

2. J. Charles et al., CKMfitter Group, Eur. Phys. J. C41, 1 (2005);

Updated results and plots at http://ckmfitter.in2p3.fr.

3. R. M. Baltrusaitis et al., Phys. Rev. Lett. 56, 2140 (1986); Adler J. et al., Phys. Rev. Lett. 60, 89 (1988).

4. D. Atwood, I. Dunietz and A. Soni, Phys. Rev. Lett. 78, 3257 (1997).

5. J.L. Rosner et al., Phys. Rev. Lett. 100, 221801 (2008); D. Asner et al., Phys. Rev. D 78, 012001 (2008).

6. D. Atwood and A. Soni, Phys Rev. D 68, 033003 (2003).

7. N. Lowrey et al., Phys. Rev. D 80, 031105 (2009).

8. Belle Collaboration, arXiv:0803.3375v1 [hepex] (2008).

9. B. Aubert et al., Phys. Rev. D 78, 034023 (2008).

10. Giri et al., Phys. Rev. D 68, 054018 (2003); Bondar et al., Eur. Phys. J. C 47, 347-353 (2006).

11. R. A. Briere et al., Phys. Rev. D 80, 032002 (2009).

12. J. Libby et al., arXiv:1010.2817 [hep-ex] (2010).

13. J. Libby, CERN-LHCb-2007-141, 2007, unpublished.

14. K. Akiba et al., CERN-LHCb-2008-031, 2008, unpublished.

15. P. Naik et al., Phys. Rev. D 80, 112004 (2009).

16. B. I. Eisenstein et al., Phys. Rev. D 78, 052003 (2008).

17. J. P. Alexander et al., Phys. Rev. D 79, 052001 (2009).

18. P. U. E. Onyisi et al., Phys. Rev. D 79, 052002 (2009).

19. D. Besson et al., Phys. Rev. D 80, 032005 (2009).

20. C. Aubin et al., Phys. Rev. Lett. 94, 011601 (2005). 

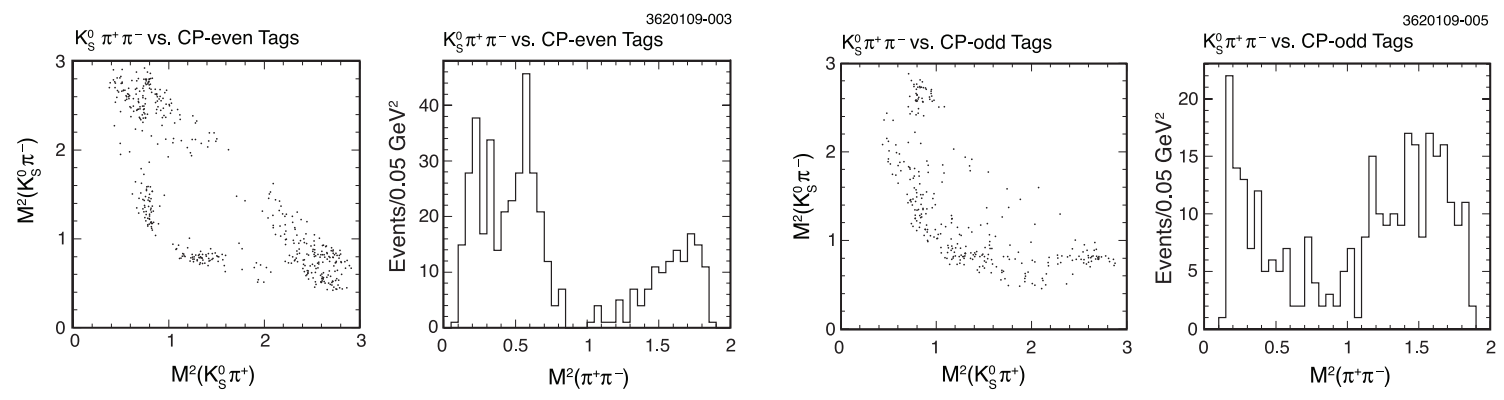

Figure 1. Effects of $C P$ correlation on the Dalitz plot of the decay $D^{0} \rightarrow K_{S}^{0} \pi^{+} \pi^{-}$. The $K_{S}^{0} \rho$ component, clearly visible when $K_{S}^{0} \pi^{+} \pi^{-}$recoils against a CP-even tag, disappears opposite a CP-odd tag.

Table 1

CLEO-c measurements of $D^{+}$and $D_{s}^{+}$leptonic decay branching fractions and decay constants.

\begin{tabular}{ll}
\hline & CLEO-c Result \\
\hline$B\left(D^{+} \rightarrow \mu^{+} \nu\right)[16]$ & $(3.82 \pm 0.32 \pm 0.09) \times 10^{-4}$ \\
$B\left(D_{s}^{+} \rightarrow \mu^{+} \nu\right)[17]$ & $(5.65 \pm 0.45 \pm 0.17) \times 10^{-3}$ \\
$B\left(D_{s}^{+} \rightarrow \tau^{+} \nu\right)\left(\right.$ from $\left.\tau^{+} \rightarrow \pi^{+} \bar{\nu}\right)[17]$ & $(6.42 \pm 0.81 \pm 0.18) \times 10^{-2}$ \\
$B\left(D_{s}^{+} \rightarrow \tau^{+} \nu\right)\left(\right.$ from $\left.\tau^{+} \rightarrow e^{+} \nu \bar{\nu}\right)[18]$ & $(5.30 \pm 0.47 \pm 0.22) \times 10^{-2}$ \\
$B\left(D_{s}^{+} \rightarrow \tau^{+} \nu\right)\left(\right.$ from $\left.\tau^{+} \rightarrow \rho^{+} \bar{\nu}\right)[15]$ & $(5.52 \pm 0.57 \pm 0.21) \times 10^{-2}$ \\
\hline$f_{D^{+}}$ & $(205.8 \pm 8.5 \pm 2.5) \mathrm{MeV}$ \\
$f_{D_{s}^{+}}$(combined) & $(259.0 \pm 6.2 \pm 3.0) \mathrm{MeV}$ \\
\hline
\end{tabular}
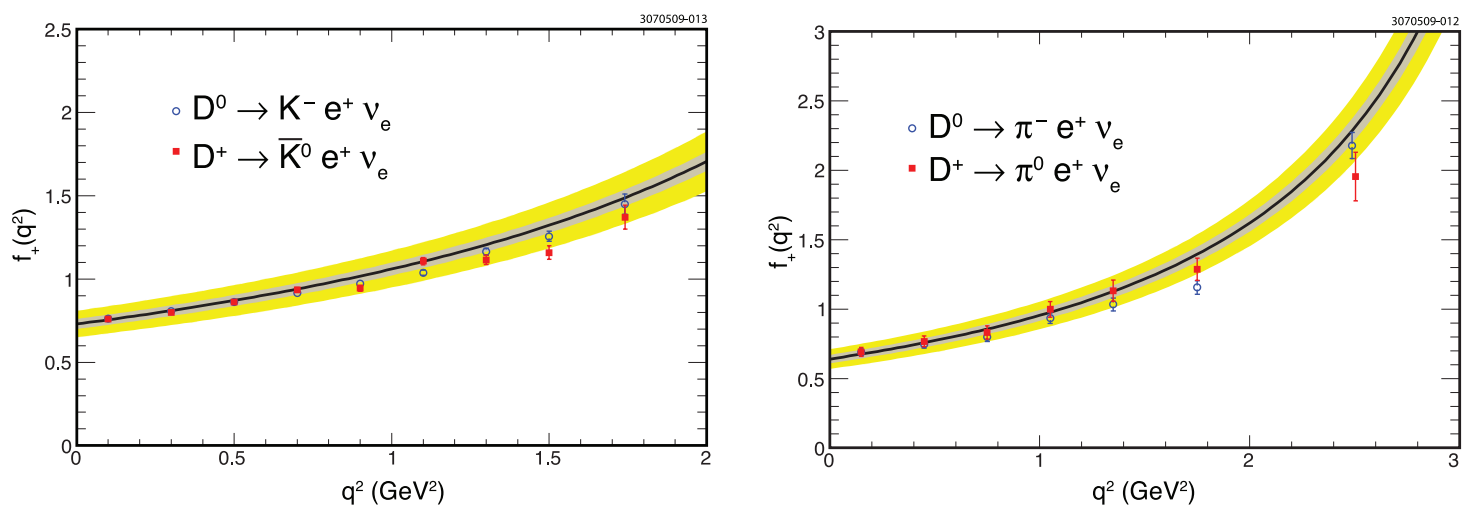

Figure 2. $D$ semileptonic decay form factors for kaon (left) and pion (right) decays as a function of $q^{2}$. The points are CLEO-c data, and the bands are from a lattice QCD prediction. 